\title{
Fissuration en relaxation des jonctions soudées en aciers inoxydables austénitiques
}

\author{
Lucien Allais ${ }^{1, a}$, Quentin Auzoux ${ }^{1}$, Magali Reytier $^{2}$ et André Pineau ${ }^{3}$ \\ 1 Commissariat à l'Énergie Atomique, Direction de l'Énergie Nucléaire, Département des Matériaux pour le Nucléaire, \\ Service de Recherches Métallurgiques Appliquées, Centre d'Étude de Saclay, 91191 Gif-sur-Yvette Cedex, France \\ 2 Commissariat à l'Énergie Atomique, Direction de l'Énergie Nucléaire, Département de Modélisation des Systèmes \\ et des Structures, Service d'Études Mécaniques et Thermiques, Centre d'Étude de Saclay, 91191 Gif-sur-Yvette Cedex, France \\ 3 UMR CNRS 7633, Centre des Matériaux, École des mines de Paris, BP 87, 91003 Évry Cedex, France
}

Reçu le 2 juillet 2004, accepté le 5 novembre 2004

Résumé - Cet article représente une synthèse des études menées au CEA/SRMA, en collaboration avec l'ENSMP, sur la fissuration en relaxation (F.E.R.) des aciers inoxydables austénitiques. Initialement, l'étude a porté sur l'endommagement des Zones Affectées (Z.A.) des soudures en aciers stabilisés au titane de type AISI 321. Une démarche de simulation expérimentale de Z.A. par des traitements thermo-mécaniques a été mise en place. Un essai de relaxation sur éprouvette CT a également été mis au point afin de reproduire en laboratoire ce type de fissuration. Outre la validation de cette démarche, il a été montré que l'écrouissage joue un rôle prépondérant sur la fragilisation intergranulaire et que le vieillissement atténue cette fragilisation contrairement à ce qui est couramment admis dans la littérature. Cette remise en cause du rôle premier de la précipitation des carbonitrures de titane permet d'élargir le champ d'investigation aux aciers non stabilisés pour lesquels il existe un certain nombre de cas de fissuration similaires à ceux attribués à la F.E.R. En appliquant la démarche précédente à des aciers de type AISI 316 et AISI 304, on montre aussi que l'écrouissage est un facteur de fragilisation intergranulaire. Parallèlement, on établit, par approche locale de la rupture, un modèle d'endommagement intergranulaire utilisable pour l'évaluation du risque de fissuration sur des composants réels.

Mots clés : Fissuration / relaxation / rupture intergranulaire / acier inoxydable austénitique / jonction soudée / approche locale

\begin{abstract}
Reheat cracking in austenitic stainless steels. Several studies concerning reheat cracking of austenitic stainless steels have been conducted in the frame of a collaboration between CEA/SRMA and ENSMP. The present paper summarizes these studies. The first study focused on a Ti-stabilized AISI 321 stainless steel. Heat affected zones (HAZ) were simulated by means of a thermo-mechanical treatment. Relaxation tests on pre-cracked CT specimens were performed in order to reproduce the reheat cracking phenomenon. Comparisons between real and simulated HAZ were made and the whole method was validated. The important role played by the strain accumulated during welding process was shown. Contrarily to the commonly assumed mechanism, aging of HAZ decreased their embrittlement. As the intragranular titanium carbides did not appear to be the major reason for the intergranular embrittlement, the same study was undertaken to investigate the reheat cracking sensitivity of unstabilized stainless steels AISI 316 and AISI 304. Pre-strain embrittlement was also noticed on these steels. Using the local approach of fracture, an intergranular damage model was developed. It provides quantitative prediction of the reheat cracking risk and can also be used to assess creep damage under multi-axial stress state.
\end{abstract}

Key words: Reheat cracking / creep / intergranular damage / austenitic stainless steel / weldment / local approach / relaxation

a Auteur correspondant : lucien.allais@cea.fr 


\section{Nomenclature}

\begin{tabular}{|ll|}
\hline$D$ & $\begin{array}{l}\text { dommage de fluage : fraction de joints de } \\
\text { grains endommagée }\end{array}$ \\
$\varepsilon_{\text {fluage }}$ & $\begin{array}{l}\text { déformation équivalente de fluage } \\
A, \alpha \text { et } \beta\end{array}$ \\
& $\begin{array}{l}\text { coefficient et exposants de la loi d'endom- } \\
\text { magement de fluage }\end{array}$ \\
$p$ et $q$ & $\begin{array}{l}\text { coefficients du modèle de Spindler pour la } \\
\text { correction du modèle de ductilité critique } \\
\text { en fluage sous contraintes multiaxiales }\end{array}$ \\
& plus grande contrainte principale \\
$\sigma_{1}$ & contrainte équivalente de von Mises \\
$\bar{\sigma}$ & contrainte hydrostatique \\
$\sigma_{\mathrm{P}}$ &
\end{tabular}

\section{Introduction}

Les aciers inoxydables austénitiques sont largement employés pour la fabrication de composants industriels qui opèrent à des températures comprises entre $450{ }^{\circ} \mathrm{C}$ et $600{ }^{\circ} \mathrm{C}$. L'expérience acquise dans le domaine des centrales nucléaires et thermiques en France comme à l'étranger a montré qu'un risque de fissuration au voisinage des jonctions soudées existait pour des durées inférieures aux durées de vie prévues.

Ce type de fissuration intergranulaire dans les zones affectées (Fig. 1) a été identifié dans les années 1950 principalement sur les aciers stabilisés au titane et niobium [1-3]. Il est connu sous les termes de « fissuration en relaxation » ou «fissuration au réchauffage » (F.E.R.). Il se dégage, des nombreuses études sur ce sujet [4], un large consensus sur le mécanisme de fragilisation intergranulaire des zones affectées. Au cours du soudage, les carbures de titane ou niobium sont remis en solution près de la ligne de fusion et cette zone subit également un écrouissage dû aux contraintes de bridage. Lors du fonctionnement à haute température, la relaxation des contraintes résiduelles induit des déformations inélastiques localisées près des jonctions soudées sièges de défauts. Par ailleurs, de fins carbonitrures de titane ou niobium secondaires précipitent sur les dislocations, qui constituent des sites de germination, comme le montre la figure 1. Les auteurs de ces études attribuent la fragilisation des joints de grains au durcissement intragranulaire provoqué par l'interaction des dislocations avec les carbonitrures secondaires.

Afin de se prémunir de ce type de fissuration des structures mécano-soudées fonctionnant dans le domaine du fluage significatif, une étude a été menée au CEA en collaboration avec l'École des mines de Paris [5] pour être en mesure de disposer d'outils d'évaluation quantitative de ce risque. Le présent article décrit de manière chronologique et synthétique cette étude qui a porté, initialement sur un acier stabilisé au titane AISI 321, puis, au regard des résultats, sur des aciers non stabilisés AISI 304 et AISI 316. Les résultats de ces travaux sont reportés en détail ailleurs [5-9]. Les compositions des matériaux étudiés sont reportées dans le tableau 1. a)

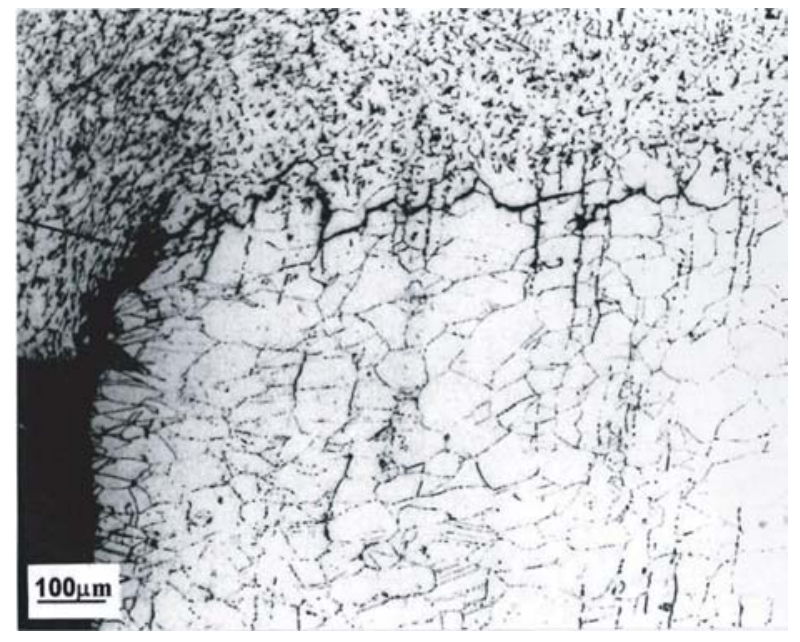

b)

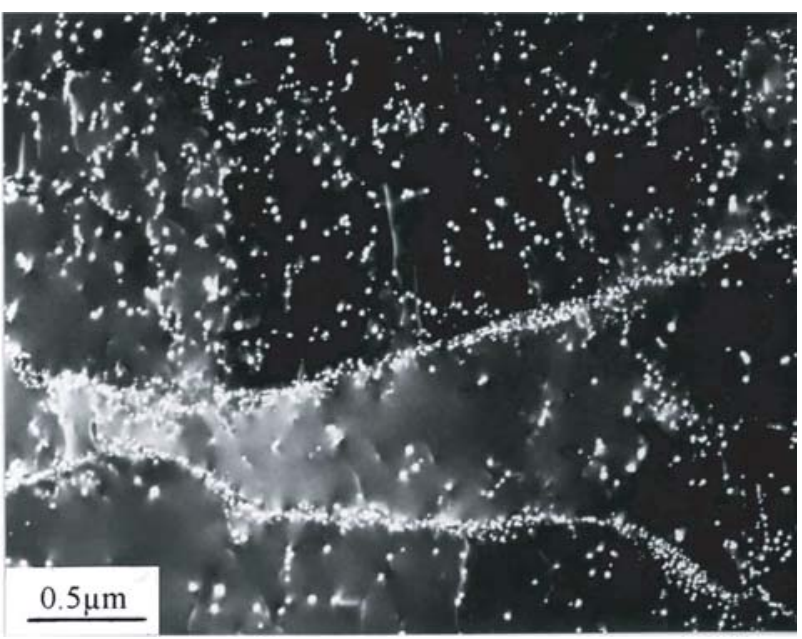

Fig. 1. a) Fissuration intergranulaire dans la Zone Affectée (Z.A.) d'une jonction soudée en acier AISI 321 et b) observation par microscopie électronique en transmission en champ sombre des précipités ( $\mathrm{TiC}$ ) secondaires dans la zone affectée.

\section{2 Étude de la fissuration en relaxation de l'acier AISI 321}

\subsection{Démarche de l'étude}

Obtenir des données quantitatives à partir de soudures réelles s'avère très difficile puisque les zones affectées où se produisent les fissures sont très petites (quelques centaines de $\mu \mathrm{m}^{3}$ ) et présentent un fort gradient de microstructure et de propriétés mécaniques. Une solution possible consiste donc à simuler ces zones affectées (Z.A.) de manière à disposer d'une quantité de matériau suffisamment importante pour pouvoir en déterminer le comportement mécanique et en étudier l'endommagement.

\subsubsection{Simulation de zones affectées}

La simulation des zones affectées doit avant tout reproduire la microstructure obtenue après soudage puis 
Tableau 1. Compositions chimiques en pourcentages massiques des aciers étudiés.

\begin{tabular}{ccccccccccccccc}
\hline Matériau & $\mathrm{N}$ & $\mathrm{C}$ & $\mathrm{Mn}$ & $\mathrm{Cr}$ & $\mathrm{Si}$ & $\mathrm{Ni}$ & $\mathrm{Mo}$ & $\mathrm{S}$ & $\mathrm{Ti}$ & $\mathrm{P}$ & $\mathrm{Co}$ & $\mathrm{Cu}$ & $\mathrm{Nb}$ & $\mathrm{B}$ \\
\hline AISI 321 & 0,014 & 0,06 & 1,63 & 18,0 & 0,49 & 10,2 & 0,23 & 0,012 & 0,56 & 0,024 & & & $<0,05$ & 0,0018 \\
AISI 304H & 0,034 & 0,059 & 1,23 & 18,4 & 0,68 & 11,8 & 0,33 & 0,03 & $<0,02$ & 0,028 & & 0,12 & $<0,05$ & $<0,0005$ \\
AISI 316L $(\mathrm{N})$ & 0,068 & 0,029 & 1,73 & 17,3 & 0,31 & 12,1 & 2,53 & 0,001 & & 0,023 & 0,089 & 0,29 & & 0,0003 \\
\hline
\end{tabular}

au cours du vieillissement en service à $550^{\circ} \mathrm{C}$. Le cycle thermique à haute température que subissent ces zones, lors du soudage, a pour principale conséquence de remettre en solution les carbonitrures de titane. Durant cette opération, ces zones sont également écrouies. Enfin, en service, ces matériaux sont soumis à un vieillissement à une température de $550{ }^{\circ} \mathrm{C}$, qui génère une précipitation secondaire de fins carbonitrures de titane.

Par conséquent, la simulation de la microstructure après soudage se fait en trois étapes successives : hypertrempe, écrouissage et vieillissement. L'hypertrempe est réalisée à une température inférieure à la température réelle mais suffisamment élevée pour pouvoir dissoudre les carbures de titane et ce pendant la durée nécessaire pour que ce traitement soit homogène sur un coupon d'une épaisseur d'une vingtaine de millimètres. L'écrouissage est réalisé par un laminage pouvant aller jusqu'à 30 pourcent de réduction d'épaisseur. Enfin, une matrice d'essais en temps (de 500 à 10000 heures) et température (de 550 à $650{ }^{\circ} \mathrm{C}$ ) est définie afin que ces Z.A. simulées subissent un vieillissement accéléré.

Comme le montre la figure 2, les microstructures obtenues sont semblables aux microstructures réelles (Fig. 1). L'analyse quantitative des histogrammes des carbonitrures de titane, reportée dans [6], montre que les Z.A. représentatives des Z.A. fissurées sont obtenues pour un taux d'écrouissage de $15 \%$. Ces traitements thermomécaniques de simulation sont donc validés et permettent de disposer de coupons de tôle de Z.A. simulées.

\subsection{2 Étude du comportement mécanique des zones affectées simulées}

L'étude détaillée du comportement de ces Z.A. simulées est décrite dans [5]. Les résultats de dureté montrent que l'écrouissage induit bien évidemment un durcissement. L'effet du vieillissement est également de durcir les Z.A. soumises à un écrouissage inférieur à $15 \%$ tandis que l'on note un adoucissement des Z.A. écrouies au-delà de ce seuil. Ces résultats sont la conséquence d'une compétition entre un durcissement par interaction dislocations-précipités et la ségrégation des éléments interstitiels qui, en interagissant avec les dislocations, sont également à l'origine d'un durcissement et un adoucissement dû à la restauration du réseau de dislocations.

L'écrouissage améliore la tenue en fluage (augmentation du temps à rupture) mais diminue la ductilité. En revanche, le vieillissement, contrairement à ce que l'on pouvait attendre, diminue la tenue en fluage en réduisant les temps à rupture et en augmentant la ductilité. La

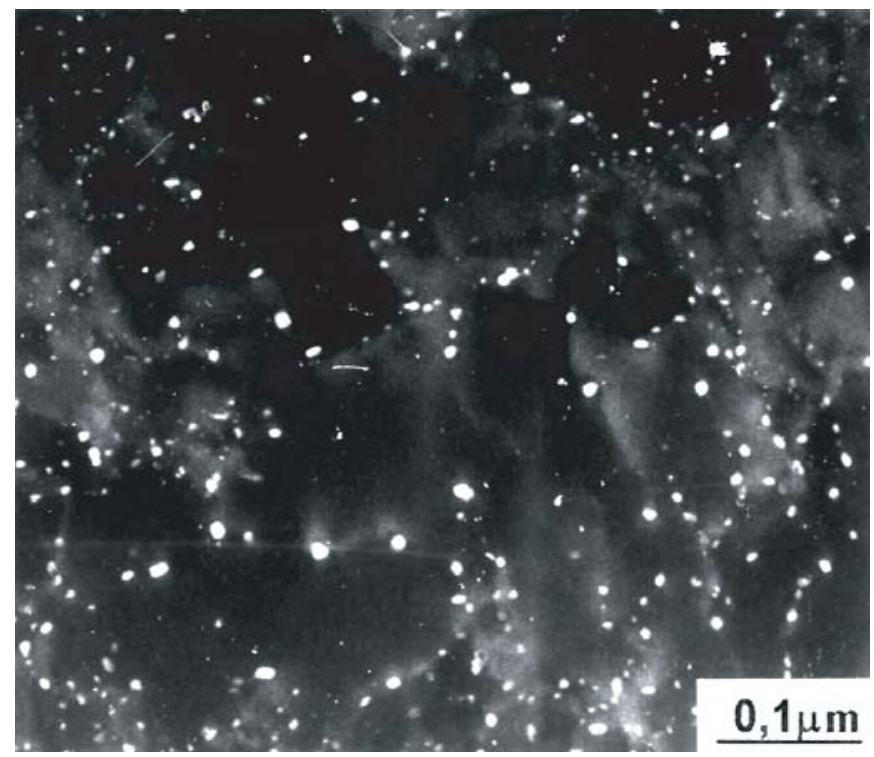

Fig. 2. Observation par microscopie électronique en champ sombre des précipités (TiC) d'une Z.A. simulée écrouie $15 \%$ et vieillie à $650{ }^{\circ} \mathrm{C}$ pendant 500 heures.

notion de durcissement intragranulaire par interactions dislocations-précipités secondaires de carbonitrure de titane est donc remise en cause.

\subsubsection{Reproduction de la fissuration en relaxation en laboratoire}

Une des étapes importantes de la démarche mise en place est de pouvoir reproduire en laboratoire ce type de fissuration. Du point de vue du chargement, il faut, tout d'abord, créer une importante quantité d'énergie élastique emmagasinée afin de reproduire l'effet des contraintes résiduelles générées par le soudage, puis, comme c'est le cas pour le composant lors de son fonctionnement, ne plus apporter d'énergie sous forme mécanique. Il faut également prendre en compte un défaut. Un des essais mécaniques qui répond à ces exigences est un essai de relaxation sur éprouvette préfissurée de type CT. Le chargement se fait jusqu'à un certain niveau d'effort pour lequel on s'assure que la fissure ne s'amorce pas, puis on maintient constante l'ouverture de l'éprouvette : l'apport d'énergie ne se fait que lors du chargement. 


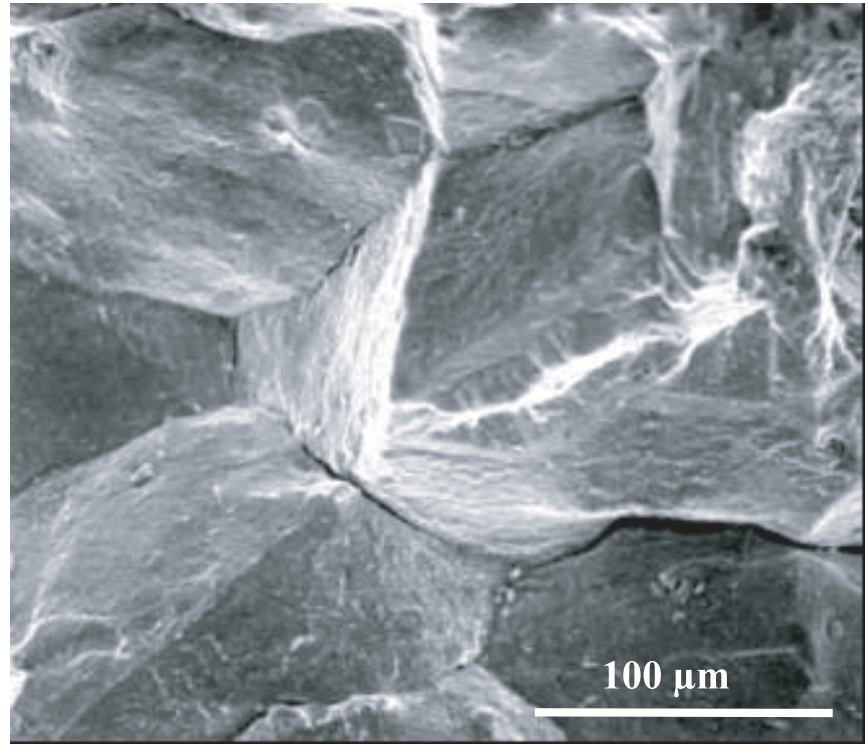

Fig. 3. Faciès de rupture intergranulaire après un essai de relaxation sur éprouvette CT en Z.A. écrouie $15 \%$ non vieillie.

Comme chaque Z.A. simulée présente un comportement mécanique différent, il n'est pas possible d'imposer la même valeur d'effort lors de la phase de chargement. Il faut donc trouver une valeur qui, par rapport à chaque comportement de Z.A. simulée, soit identique. À cet effet, on effectue un essai préliminaire afin d'établir la courbe effort-ouverture sur laquelle on détermine la charge correspondant à l'écart à la linéarité. C'est cette valeur qui est choisie comme étant le seuil de chargement de l'essai de relaxation.

Pour toutes les Z.A. simulées, on réalise ce type d'essai à $600{ }^{\circ} \mathrm{C}$ et ce pour une durée d'environ 1000 heures. Après essai, on détermine si la fissure s'est amorcée et l'on mesure la longueur de l'éventuelle propagation.

Les essais de relaxation sur éprouvette CT sur métal de base ne conduisent jamais à l'amorçage de la fissure par relaxation; les déformations inélastiques permettant de dissiper l'énergie élastique emmagasinée. On constate, en revanche, que toutes les Z.A. simulées écrouies de $10 \%$ ou plus présentent une propagation intergranulaire après 1000 heures de relaxation, comme le montre la figure 3. Ces propagations témoignent de la fragilisation intergranulaire de l'acier due au soudage. Par ailleurs, pour un même taux d'écrouissage conduisant à une propagation intergranulaire, on remarque que plus la Z.A. est vieillie moins la longueur de propagation est importante. L'ensemble de ces résultats permet d'établir un diagramme de sensibilité à la fissuration intergranulaire en fonction du taux d'écrouissage et du degré de vieillissement. Sur ce diagramme reporté sur la figure 4 , on remarque que le domaine de fissuration est délimité par un taux d'écrouissage compris entre 5 et $10 \%$.

Afin de mettre en évidence un éventuel effet de l'environnement, un essai a été réalisé sous vide : la propagation s'est avérée similaire à celle de l'essai conduit sous air.

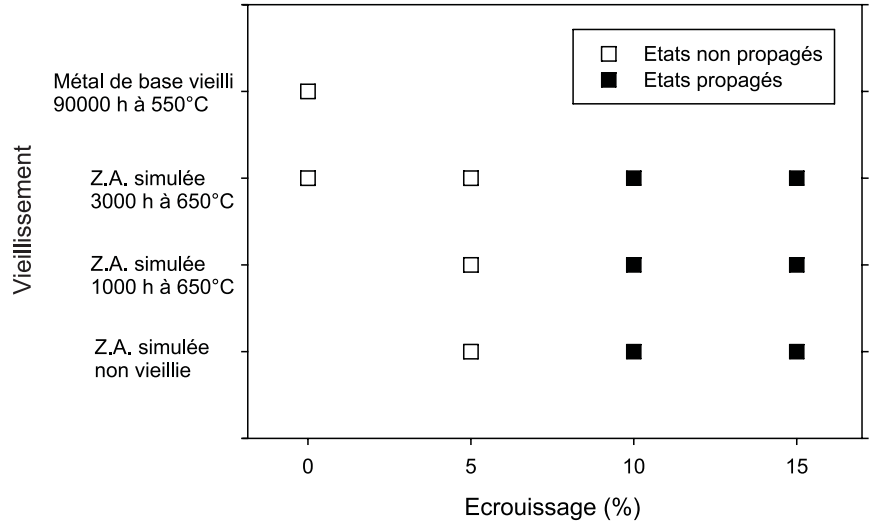

Fig. 4. Diagramme de sensibilité à la fissuration intergranulaire à $600{ }^{\circ} \mathrm{C}$ déterminé à partir des essais de relaxation sur éprouvette $\mathrm{CT}$.

\subsection{Conclusions de l'étude sur la fissuration en relaxation de l'acier AISI 321}

Grâce à la démarche de simulation de Z.A. et de reproduction du phénomène de fissuration par l'essai de relaxation sur éprouvette CT, il a été possible de mettre en évidence la fragilisation intergranulaire propre aux microstructures représentatives des zones situées près de la soudure. En outre, les résultats de propagation intergranulaire montrent clairement que le taux d'écrouissage est le paramètre prépondérant qui induit cette fragilisation. Le vieillissement semble diminuer la sensibilité à la fissuration intergranulaire, puisqu'à taux d'écrouissage fixé, les longueurs de propagation diminuent avec la durée de vieillissement. Ce résultat est en contradiction avec les interprétations de la littérature $[1,3]$ qui indiquent que le vieillissement favorise ce type de fissuration. Ces interprétations reposent sur le fait qu'il existe un durcissement intragranulaire dû à l'interaction dislocations-précipités secondaire. Cependant, l'étude du comportement en fluage des Z.A. simulées montre que le vieillissement, au lieu d'augmenter la tenue en fluage, conduit à une accélération de la vitesse de fluage traduisant, un adoucissement intragranulaire. D'un point de vue expérimental, il n'existe pas de contradiction, car le durcissement intragranulaire dû au vieillissement n'a été mis en évidence qu'à température ambiante. Il est à noter que cette tendance a déjà été relevée expérimentalement dans [10] mais n'a jamais été utilisée pour modifier l'interprétation du mécanisme de F.E.R.

En définitive, la conclusion de cette étude [5] sur le mécanisme responsable de la fragilisation intergranulaire est très similaire à celle des précédents travaux, à savoir que c'est un durcissement intragranulaire qui est à la base de ce phénomène de fissuration. En revanche, ce durcissement est maximal en début de maintien en température, puisqu'il est dû à l'écrouissage produit par le soudage et est renforcé par les interactions du réseau de dislocations avec les éléments remis en solution (carbone, azote et titane) lors du soudage. Lors du fonctionnement en service en température, la restauration du réseau de 


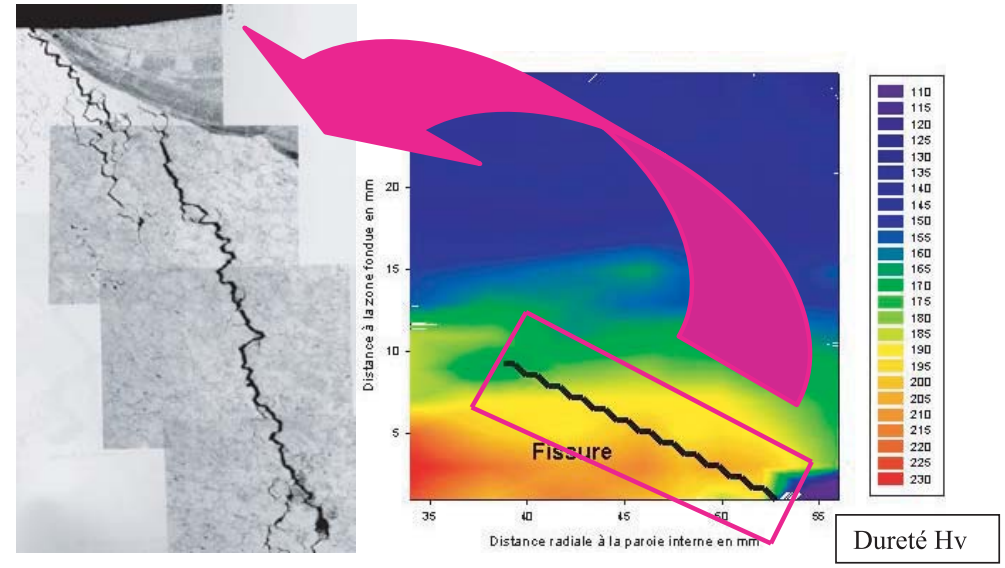

Fig. 5. Cas d'une fissuration intergranulaire observée sur un composant en acier AISI AISI 304H et cartographie de dureté Hv de la zone affectée.

dislocations et la disparition de ces éléments en solution qui reprécipitent conduisent à un adoucissement intragranulaire et donc à une diminution de la sensibilité à la fissuration intergranulaire.

Du point de vue industriel, les principales répercussions de ce constat sont, d'une part, que le risque de fissuration est maximal en début de vie du composant mécano-soudé et, d'autre part, que les carbonitrures secondaires de titane n'étant pas un élément indispensable à ce phénomène, il est légitime de poser la question de l'existence de ce phénomène pour les aciers inoxydables austénitiques de la série 300 non stabilisés.

\section{3 Étude de la fissuration en relaxation sur aciers non stabilisés}

La fragilisation éventuelle des aciers non stabilisés est d'autant plus intéressante à étudier que quelques cas de fissuration intergranulaire concernant ces aciers ont été rapportés [11,12]. Ces cas présentent les caractéristiques de la F.E.R., à savoir une fissure intergranulaire confinée dans la zone affectée (Fig. 5) et des chargements en service trop faibles pour expliquer cet endommagement. Un second travail de thèse [9] a donc été engagé en considérant les aciers AISI 304H, AISI 316H et AISI 316L(N). Pour ces aciers, contrairement aux Z.A. réelles en acier AISI 321 déposées au bout de 90000 heures à $550{ }^{\circ} \mathrm{C}$ et donc très affectées par cette durée (restauration presque complète du réseau de dislocations créé lors du soudage), au niveau de la soudure, le champ de dureté de la Z.A. fissurée en AISI 304H (Fig. 5) met en évidence un gradient de propriété mécanique dû à l'écrouissage généré par le soudage.

\subsection{Simulation de zones affectées}

Étant donné la disponibilité de soudures neuves d'acier AISI $316 \mathrm{~L}(\mathrm{~N})$ et connaissant le rôle secondaire du vieillissement, il est essentiel que les Z.A. simulées soient représentatives des soudures non vieillies. Les filiations de dureté font apparaître que la zone affectée s'étend sur une dizaine de millimètres et présente une dureté maximale d'environ $210 \mathrm{Hv}$. Cette valeur de dureté constitue un paramètre supplémentaire pour caler le taux d'écrouissage des Z.A. simulées qui, pour cette seconde étude est réalisé à une température comprise entre 400 et $600{ }^{\circ} \mathrm{C}$. Le taux choisi est de $15 \%$ qui induit une dureté de $220 \mathrm{Hv}$. En outre, les observations par microscopie en transmission des Z.A. réelles et simulées [9] ont montré que les structures et les densités de dislocations sont similaires. Aucun traitement de vieillissement n'est appliqué pour ces Z.A. simulées qui sont donc soit non écrouies ou écrouies à un taux de $15 \%$. Cette démarche a aussi été adoptée sur un acier AISI $316 \mathrm{H}$.

\subsection{Reproduction du mécanisme}

De même que pour les Z.A. en acier AISI 321, des essais de relaxation sur éprouvette CT de Z.A. simulées en acier AISI $316 \mathrm{~L}(\mathrm{~N})$ et AISI $316 \mathrm{H}$ sont réalisés à $600{ }^{\circ} \mathrm{C}$ avec le même protocole expérimental. Pour ces deux aciers, la Z.A. écrouie conduit à une propagation intergranulaire de plusieurs millimètres après 1000 heures d'essai. En revanche, pour la Z.A. non écrouie, on ne constate jamais l'amorçage de la fissuration, même pour des chargements élevés.

\section{3 Étude du mécanisme}

Les essais de fluage sur éprouvettes lisses et entaillées ont mis en évidence une fragilisation intergranulaire par écrouissage. L'étude du mécanisme d'endommagement intergranulaire montre que, qualitativement, il n'existe pas de différence entre les matériaux écroui et non écroui. Dans les deux cas, les cavités apparaissent préférentiellement au voisinage des points triples autour des carbures intergranulaires, puis coalescent pour former des microfissures intergranulaires. À l'avant de ces microfissures, de nouvelles cavités se forment ensuite, ce qui entraîne la croissance de celles-ci, comme on peut le voir sur 


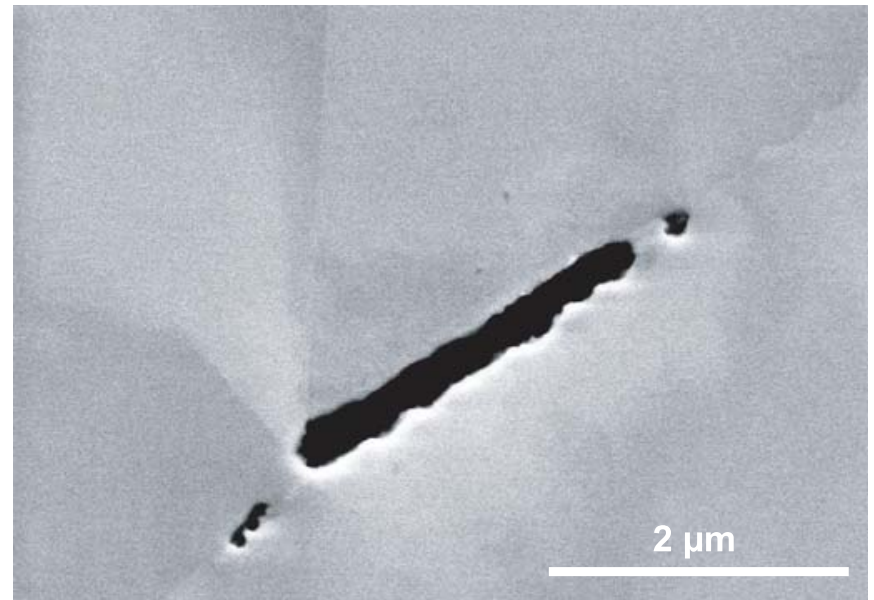

Fig. 6. Germination de cavités autour de carbures en avant d'une microfissure intergranulaire dans une Z.A. simulée AISI $316 \mathrm{~L}(\mathrm{~N})$ écrouie $15 \%$ et non vieillie.

la figure 6. La germination de l'endommagement est donc continue et contrôle la durée de vie. Du fait des incompatibilités de déformation entre grains, l'écrouissage génère des contraintes internes qui favorisent sans doute la germination, puisqu'il est établi que la germination nécessite un niveau de contrainte locale élevé [13].

\section{Modélisation de l'endommagement}

Du point de vue de l'exploitation en service ou de la conception, il est essentiel de disposer d'outils d'évaluation quantitatifs du risque de F.E.R. À cet effet, une approche locale de la rupture appliquée à l'endommagement intergranulaire a été mise en œuvre. Cette démarche est décrite en détail dans [7].

\subsection{Identification du modèle d'endommagement}

Le modèle retenu est celui développé par l'ENSMP [14] qui considère comme paramètre d'endommagement intergranulaire, $D$, la fraction de joints de grains endommagés. Comme le montre l'équation (1), il s'agit d'une formulation incrémentale qui, du point de vue mécanique, fait intervenir la déformation de fluage, $\varepsilon_{\text {fluage }}$ et la plus grande contrainte principale, $\sigma_{1}$

$$
\mathrm{d} D=A\left|\sigma_{1}\right|^{\alpha}\left(\varepsilon_{\text {fluage }}\right)^{-\beta} \mathrm{d} \varepsilon_{\text {fluage }}
$$

L'identification des coefficients de ce modèle se fait par comparaison des prévisions de valeurs de fraction de joints endommagés avec des valeurs expérimentales mesurées sur des éprouvettes sollicitées en fluage. Afin de faire varier l'état mécanique local, des essais de fluage sont menés sur des éprouvettes axisymétriques entaillées avec plusieurs rayons de fond d'entaille qui permettent d'obtenir ainsi des valeurs différentes du taux de triaxialité des contraintes. Ce type de géométrie permet l'identification du modèle sur une vaste plage d'états mécaniques mais, du fait de l'hétérogénéité de ces états dans la partie utile de ce type d'éprouvette, nécessite de faire des calculs par éléments finis [15] pour la détermination des niveaux de contraintes et déformations en tous points.

Les essais de fluage sont réalisés sur deux géométries d'éprouvettes axisymétriques entaillées ayant en commun un diamètre minimal de $6 \mathrm{~mm}$ et un rayon à fond d'entaille de 1 et $4 \mathrm{~mm}$ (respectivement, éprouvettes FLE1-6 et FLE4-6). Ces essais, menés à effort constant, sont instrumentés avec deux extensomètres permettant d'obtenir l'allongement axial de la partie utile et la déformation diamétrale moyenne de la section minimale. Pour une même charge, plusieurs essais sont réalisés mais interrompus à différents instants : les éprouvettes sont alors découpées suivant un plan médian dans lequel le dommage intergranulaire est mesuré par métallographie quantitative. En superposant le maillage du calcul par éléments finis, il est possible de mesurer le dommage pour chaque maille.

Des lois de fluage prenant en compte les stades primaire et secondaire sont déterminées à partir d'essais de fluage sur éprouvettes lisses. Les états mécaniques dans la partie utile des éprouvettes FLE sont calculés par éléments finis en utilisant ces lois. Afin de valider ces calculs, les réponses calculées globales sont comparées aux variations expérimentales des allongements axiaux et des déformations diamétrales moyennes. Pour l'éprouvette FLE4-6, ces calculs montrent une redistribution rapide de la plus grande contrainte principale, qui devient maximale au centre de l'éprouvette, tandis que pour l'éprouvette FLE1-6, cette redistribution est plus lente et conduit, au moment de la rupture, à un maximum situé entre le centre de l'éprouvette et le fond d'entaille. La répartition de la déformation équivalente de fluage est homogène dans la section minimale pour les FLE4-6, tandis qu'elle présente un maximum en fond d'entaille pour les FLE1-6.

En intégrant le modèle d'endommagement avec des coefficients présupposés, jusqu'au temps d'arrêt de l'essai, il est possible d'obtenir la valeur du dommage $D$ en tous points de l'éprouvette. Pour chaque élément du calcul par éléments finis, on dispose ainsi des valeurs de dommage mesurées et calculées : l'identification des coefficients du modèle se fait par minimisation du résidu $R$ (Éq. (2)) entre ces deux quantités.

$$
R=\sum_{i=1}^{m}\left(D_{\text {calculé }}^{i}-D_{\text {mesuré }}^{i}\right)^{2}
$$

avec $m$ le nombre d'éléments.

La figure 7 permet de visualiser, pour les deux types d'éprouvette entaillée, les prédictions de zones endommagées qui correspondent bien avec les localisations de l'endommagement mesuré. En outre, cette comparaison indique que la valeur du dommage critique, $D_{\mathrm{c}}$, qui correspond à une zone très fortement endommagée, est d'environ $2 \%$. Pour la Z.A. simulée écrouie à $15 \%$ et non vieillie, la valeur du coefficient $\alpha$ du modèle d'endommagement est de 7,5 à comparer à celui du AISI $316 \mathrm{~L}(\mathrm{~N})$ non écroui qui est de 2 . La très forte sensibilité à la fissuration 


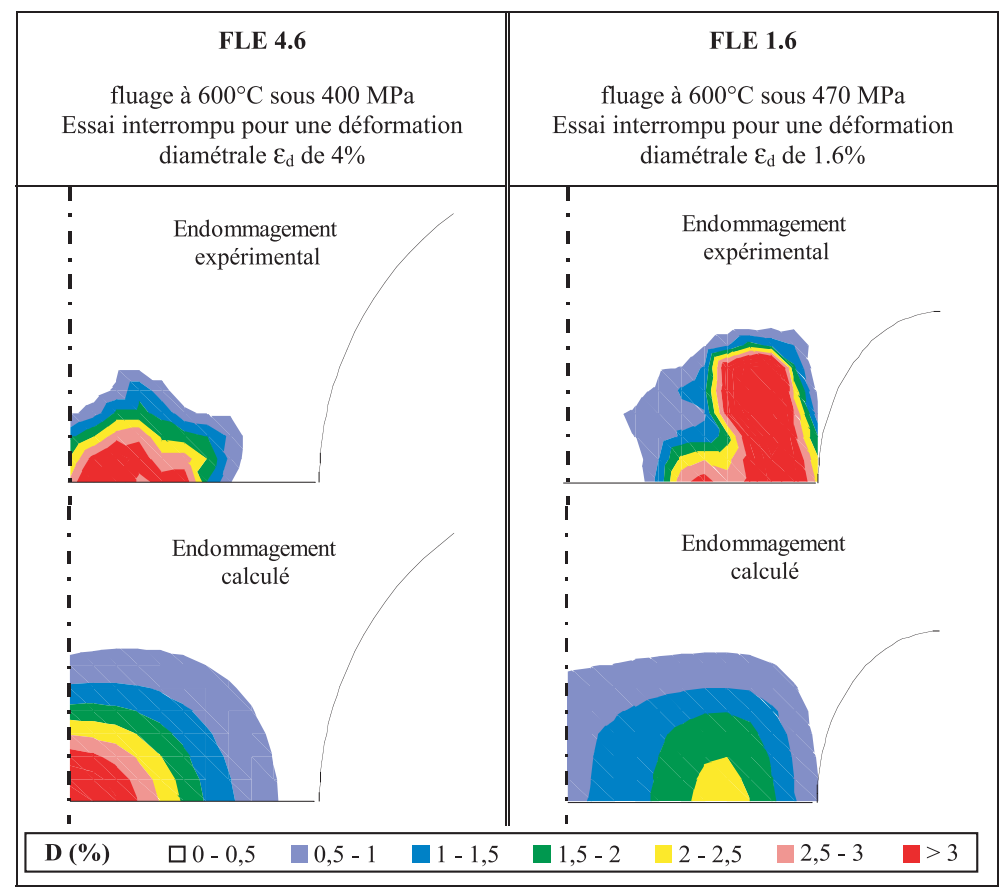

Fig. 7. Comparaison des endommagements mesurés et calculés sur une demie éprouvette FLE en Z.A. simulée AISI 321 écrouie $15 \%$ et vieillie $1000 \mathrm{~h}$ à $650^{\circ} \mathrm{C}$.

intergranulaire de cette Z.A. simulée se traduit donc par un coefficient de sensibilité à la plus grande contrainte principale élevé. La même Z.A. simulée qui a subi un vieillissement et qui est donc moins sensible voit ce coefficient diminuer à 6 .

\subsection{Validation du modèle d'endommagement}

La validation du modèle [8] repose sur son application au cas des essais de relaxation sur éprouvettes CT pour deux Z.A. simulées, l'une ayant conduit à de la propagation intergranulaire et l'autre non. Il s'agit des Z.A. simulées écrouie à $15 \%$ et non vieillie et écrouie à $5 \%$ puis vieillie à $650{ }^{\circ} \mathrm{C}$ pendant 3000 heures.

Les essais de relaxation sur éprouvettes CT sont simulés par un calcul par éléments finis $2 \mathrm{D}$ en supposant un état de contraintes planes [14]. La validation de ce calcul se fait par comparaison des courbes effort-déplacement expérimentale et calculée. La valeur de dommage calculée est obtenue par intégration au cours du temps. La prédiction de l'amorçage est établie par un niveau de dommage supérieur à la valeur du dommage critique à une distance de $150 \mu \mathrm{m}$ de la pointe de la fissure, ce qui correspond à la rupture d'un grain.

Les résultats des prévisions obtenues grâce à ces calculs sont effectivement en bon accord avec les faits expérimentaux. En outre, pour la Z.A. écrouie non vieillie, le modèle prévoit un amorçage en tout début d'essai de relaxation, ce qui a conduit à refaire l'essai et à l'interrompre après seulement 24 heures. L'examen du faciès de rupture montre que la longueur propagée après cette courte durée est similaire à celle obtenue après
1000 heures. De plus, des calculs simples montrent que les brusques diminutions de l'effort constatées en début d'essai correspondent bien à l'effet de l'avancée de fissure. La forte valeur de la plus grande contrainte principale en fond de fissure génère un dommage important mais la relaxation de l'effort due à l'avancée de fissure, associée à la forte non linéarité du modèle d'endommagement, conduit à une faible variation du dommage après un certain temps.

Un point supplémentaire de validation consiste à appliquer le modèle à un essai de fluage sur éprouvette tubulaire renfermant une entaille perpendiculaire à son axe et soumise à un état de torsion pure. Ce type d'essai, décrit en détail dans [16], présente la particularité de pouvoir séparer l'effet de la déformation de fluage et celui de la plus grande contrainte principale sur l'endommagement intergranulaire. En effet, en pointe de fissure, la déformation de fluage est maximale dans l'axe de la fissure tandis que le maximum de la plus grande contrainte principale est situé à un angle d'environ $60^{\circ}$ par rapport à l'axe du défaut. Comme on peut le remarquer sur la figure 8 , l'observation du défaut après un essai à $600{ }^{\circ} \mathrm{C}$ sur la Z.A. simulée écrouie de $15 \%$ non vieillie montre qu'il y a eu amorçage et que la direction de la fissure intergranulaire est d'environ $45^{\circ}$ par rapport à l'axe du défaut initial mettant ainsi en évidence de manière expérimentale la prépondérance de l'influence de la plus grande contrainte principale sur l'endommagement intergranulaire.

L'application du modèle est faite au moyen d'un modèle par éléments finis tridimensionnel. Le champ de dommage calculé représenté sur la figure 8b montre qu'en pointe de fissure, le maximum est effectivement localisé à un angle de $60^{\circ}$ par rapport à l'axe du défaut initial et 


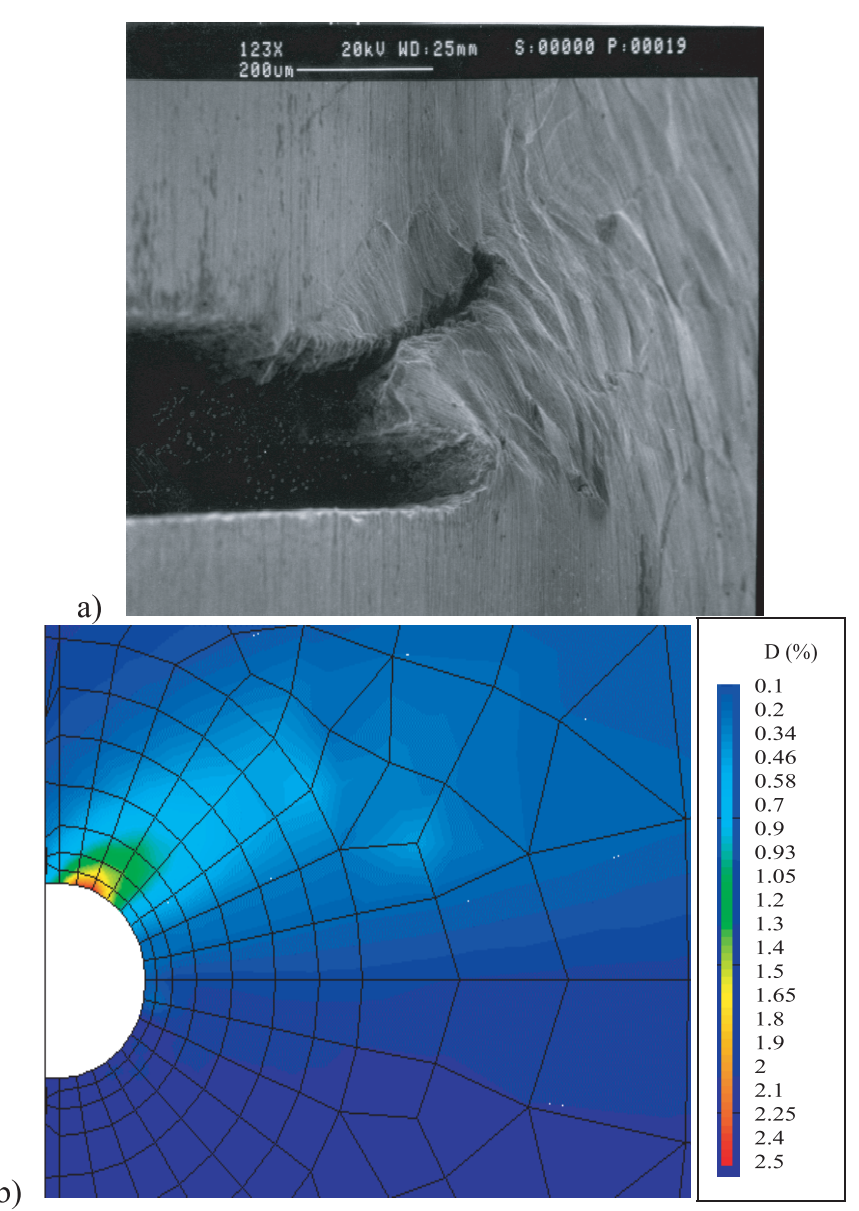

Fig. 8. Comparaison de la localisation de l'endommagement réel (a) et prédit par le modèle (b) pour un essai de fluage sur tube entaillé sollicité en torsion pure.

donc permet bien de prédire le lieu de l'amorçage intergranulaire. L'écart d'angle constaté entre l'amorçage réel et le calcul peut provenir du fait que, comme on peut le deviner sur la figure 8, la taille de grain n'est pas négligeable devant le rayon de fond d'entaille. Or la cristallographie n'est pas prise en compte par la modélisation.

\subsection{Remarques sur la modélisation de l'endommagement de fluage}

Afin de déterminer la durée de vie en fluage, certains codes de dimensionnement comme la règle R5 [17] considèrent une approche en « épuisement de la ductilité ».

La ductilité critique est assimilée à l'allongement à rupture obtenu lors d'essais de fluage uniaxiaux. Il nous semble important de rappeler que lors de ce type d'essais, la rupture n'est pas purement intergranulaire. Comme en témoignent les faciès de rupture, un endommagement de type transgranulaire ductile se développe en fin d'essai, lorsque la vitesse de déformation augmente fortement [5]. Assimiler la ductilité critique de fluage à l'allongement à rupture revient donc à intégrer la déchirure ductile qui produit la rupture effective de l'éprouvette mais qui apparaît après l'endommagement intergranulaire. Ainsi la durée de vie estimée en utilisant cette ductilité critique pourrait se révéler supérieure à la durée de vie effective si le composant rompt de manière purement intergranulaire. Il serait alors plus judicieux de choisir comme ductilité critique l'allongement de fin de stade secondaire, ou d'utiliser une approche de type Monkmann-Grant (voir par exemple [18]).

Ce type de rupture peut intervenir lorsque l'état mécanique est fortement triaxial [5]. Un nouveau modèle proposé par Spindler [19] est d'ailleurs intégré dans la règle $\mathrm{R} 5$ pour tenir compte de cet effet. Ce modèle propose la correction donnée par l'équation (3) :

$$
\begin{aligned}
& \bar{\varepsilon}_{\text {critique fluage multiaxial }}= \\
& \varepsilon_{\text {fluage uniaxial }} \exp \left[p\left(1-\frac{\sigma_{1}}{\bar{\sigma}}\right)+q\left(\frac{1}{2}-\frac{3 \sigma_{\mathrm{p}}}{2 \bar{\sigma}}\right)\right]
\end{aligned}
$$

où interviennent le taux de triaxialité défini par le rapport $\sigma_{\mathrm{p}} / \bar{\sigma}$, la plus grande contrainte principale $\sigma_{1}$ ainsi que deux coefficients ajustables, $p$ et $q$. Pour déterminer ces coefficients, Spindler a considéré les nombreux résultats d'essais de fluage en conditions biaxiales (traction/pression interne et traction/torsion) disponibles. Les valeurs prises en compte correspondent au dernier point enregistré et donc, comme pour le cas uniaxial, il est légitime de remettre en question sa pertinence lorsque la méthode est appliquée à des cas fortement triaxiaux. En effet, dans le cas des éprouvettes tubulaires, la ductilité à rupture peut être caractéristique de la phase de déchirure ductile finale, voire du flambement de l'éprouvette qui apparaît et non de l'endommagement intergranulaire.

Afin de mettre en évidence la dépendance de la ductilité de fluage avec le taux de triaxialité des contraintes, il nous a paru intéressant de fournir une meilleure estimation de celle-ci en nous appuyant sur des mesures d'endommagement intergranulaire de fluage et ce à partir des essais de fluage sur éprouvettes lisses et entaillées. Sur éprouvette lisse, pour ne pas comptabiliser le surplus de déformation dû à la déchirure ductile finale, une mesure de déformation diamétrale est réalisée en deçà de la surface de rupture et, en faisant une hypothèse de déformation à volume constant, il est possible de déterminer la déformation axiale en fin de phase d'endommagement intergranulaire avant que la déchirure ductile n'ait pris le relais. Pour les éprouvettes axisymétriques entaillées, chaque élément du maillage servant aux calculs par éléments finis est considéré comme une éprouvette indépendante ayant un état mécanique déterminé par le calcul aux éléments finis. Avec le modèle de dommage (Éq. (1)), on détermine par calcul la valeur de la déformation équivalente de fluage au sens de Von Mises qui est nécessaire pour atteindre la valeur critique du dommage intergranulaire. On peut alors estimer, en fonction du taux de triaxialité des contraintes, la variation de la ductilité pour une rupture purement intergranulaire. La figure 9 montre que la ductilité de fluage diminue très fortement lorsque le taux de triaxialité augmente. Cette 


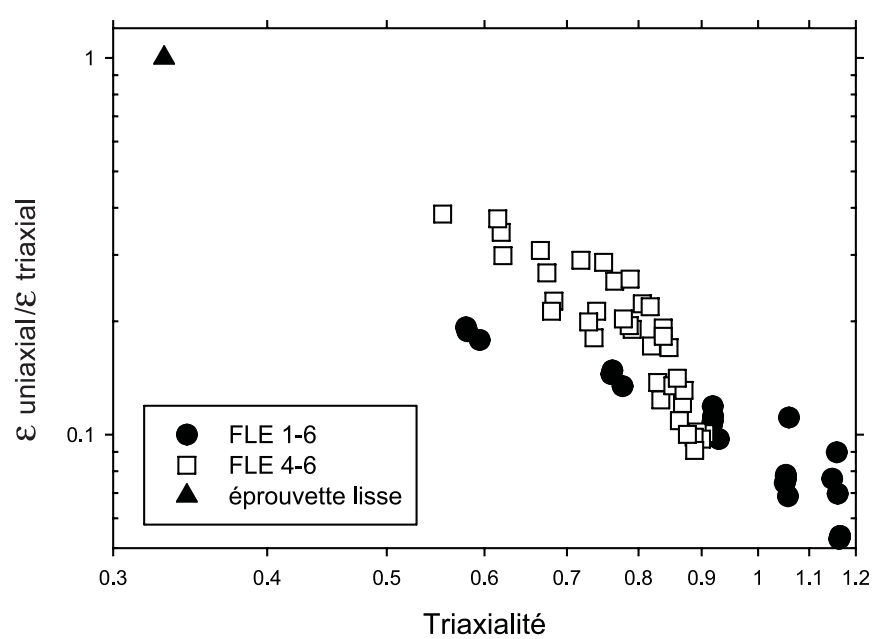

Fig. 9. Ductilité critique de dommage intergranulaire en fonction de la triaxialité des contraintes.

tendance est en accord avec les mécanismes d'endommagement intergranulaire faisant intervenir la diffusion assistée par la contrainte normale aux joints de grains [13]. Étonnamment, on peut remarquer que l'approche microscopique développée ici sur un acier AISI 321 conduit à des résultats tout à fait cohérents avec ceux obtenus par Spindler [19] qui a suivi la démarche macroscopique que nous avons décrite ci-dessus et l'a appliquée à un acier AISI $316 \mathrm{H}$.

\section{Conclusion}

En se basant sur l'explication habituellement donnée du phénomène de la F.E.R. observée sur les aciers inoxydables austénitiques stabilisés, l'étude menée au CEA avait initialement pour objectif de fournir une modélisation quantitative du risque de ce type de fissuration. À cet effet, une démarche de simulation de Z.A. a été mise en place afin d'être en mesure de travailler sur des volumes suffisants en vue d'obtenir des données quantitatives. Cette démarche a été validée tant par la représentativité des Z.A. simulées que par la reproduction, en laboratoire, du mécanisme de rupture lors d'un essai de relaxation sur éprouvette préfissurée du type CT. Cette campagne d'essais a permis de mettre en évidence le rôle prépondérant de l'écrouissage sur la fragilisation intergranulaire et un effet contraire du vieillissement. Suite à celui-ci, le durcissement par interaction des dislocations avec les précipités secondaires auquel est attribuée habituellement cette fragilisation n'est pas effectif puisqu'au contraire, le comportement en fluage axial montre que le vieillissement induit un adoucissement. L'origine de cet adoucissement est liée à la diminution de la teneur des éléments en solution solide, suite à la précipitation des carbures secondaires.
Ces conclusions remettent en question le rôle des éléments stabilisants au travers de leurs précipités secondaires sur la F.E.R. et autorisent une ouverture de ce type de fissuration aux aciers non stabilisés de la série 300. Ceci est d'autant plus intéressant qu'il existe quelques cas de fissuration intergranulaire inexpliqués et présentant les mêmes caractéristiques que ceux de la F.E.R. Une démarche analogue conduite actuellement sur des aciers AISI $316 \mathrm{~L}(\mathrm{~N})$ et AISI $316 \mathrm{H}$ met également en évidence la fragilisation intergranulaire générée par l'écrouissage.

L'identification, par approche locale de la rupture, d'un modèle d'endommagement validé permet d'évaluer le risque de fissuration sur composant. En effet, disposant de modèles de comportement et d'endommagement sur le métal de base et plusieurs types de Z.A., il est possible de déterminer l'état mécanique en tout point du composant et, à partir de ces résultats, d'évaluer la prévision de dommage. Ce modèle rend notamment compte de la chute de la ductilité de fluage lorsque le taux de la triaxialité des contraintes augmente.

\section{Références}

[1] C.F. Meitzner, Stress relief cracking in steel weldments, Welding Research Council Bulletin 211 (1975) 1-17

[2] C. Picker, Materials and structural integrity experience from PFR, Nuclear Energy 31 (1992) 207-219

[3] R.N. Younger, R.G. Baker, Heat-affected zone cracking in welded austenitic steels during heat treatment, British welding J. (1961) 579-587

[4] A. Dhooge, Survey on reheat cracking in austenitic stainless steels and Ni base alloys, Welding in the world 41 (1998) 206-219

[5] M. Chabaud-Reytier, Étude de la fissuration différée par relaxation d'un acier inoxydable austénitique stabilisé au titane, Thèse ENSMP, 1999

[6] M. Chabaud-Reytier, L. Allais, D. Dubuisson, C. CäesHogrel, A. Pineau, Mechanisms of stress relief cracking in titanium stabilised austenitic stainless steel, J. Nuclear Materials 323 (2003) 123-137

[7] M. Chabaud-Reytier, L. Allais, D. Poquillon, C. CäesHogrel, M. Mottot, A. Pineau, Modelling creep damage in heat affected zone in 321 stainless steel, Part I : quantitative study of intergranular damage, Materials at high temperatures 18(2) (2001) 71-80

[8] D. Poquillon, M. Chabaud-Reytier, L. Allais, A. Pineau, Modelling creep damage in heat affected zone in 321 stainless steel, Part II, Application to creep crack initiation simulations, Materials at high temperatures 18(2) (2001) 82-90

[9] Q. Auzoux, L. Allais, A.F. Gourgues, A. Pineau, Reheat cracking in austenitic stainless steels, Proceedings of the 14th Biennal European Conference on Fracture, ECF 14 Cracovie, 8-13 Sep. I 2002, pp. 137-144

[10] J.D. Cook, D.R. Harries, A.C. Roberts, Some factors affecting the creep strength of $20 \% \mathrm{Cr}-25 \% \mathrm{Ni}-\mathrm{Nb}$ austenitic steel at $750{ }^{\circ} \mathrm{C}$, Proceedings of the conference on creep strength in steels and high temperature alloys, Sheffield, 1972, pp. 91-98 
[11] M.T. Cabrillat, P. Allègre, E. Pluyette, B. Michel, Intergranular reheat cracking in $304 \mathrm{H}$ components. Experiments and damage evaluation, in Structural Mechanics In Reactor Technology 16, Washington 2001, paper LW4

[12] M.C. Coleman, D.A. Miller, R.A. Stevens, Reheat cracking and strategies to assure integrity of type 316 welded components, in Integrity of High-Temperature Welds, Conference, Nottingham, November, Professional Engineering Publishing Ltd., Bury St Edmunds, IP32 6BW, UK, 1998, pp. 169-179

[13] H. Riedel, Fracture at high temperatures, Springer Verlag, 1987

[14] M. Yoshida, C. Levaillant, R. Piques, A. Pineau, Quantitative study of intergranular damage in an austenitic stainless steel on smooth and notched bars, High temperature fracture mechanisms and mechanics, P. Bensussan, J.P. Mascarel (eds.), MEP, 1990, pp. 3-21
[15] CASTEM 2000, CEA Finite Element Code (http://www.cast3m.cea.fr/cast3m/index.jsp)

[16] D. Poquillon, M.T. Cabrillat, A. Pineau, Mode II creep crack initiation in $316 \mathrm{~L}(\mathrm{~N})$ stainless steel. Experiment and modelling, Materials at high temperatures 16(2) (1999) 99-107

[17] R5. Assessment Procedure for the High Temperature Response of Structures, Bristish Energy, Gloucester, UK 2001

[18] F. Garofalo, Creep rupture behaviour of notched and unotched specimens of type 304, 316 and 321 austenitic stainless steels, Proc. ASTM 159, pp. 957-972

[19] M.W. Spindler, The multiaxial creep ductility of austenitic stainless steels, in: High Temperature Mechanical Testing Committee Meeting on Multiaxial Creep Testing and Interpretation, Institute of metals, London, 2001 